\title{
Evaluation of Plasma D-Dimer Concentration Among Normal and Complicated Pregnancies, Saudi Arabia
}

\author{
Rana Zaini $^{\mathbb{1}}$, Amani Al-Rehaili ${ }^{*}{ }^{\mathbb{D}}$, Rahma Kufia $^{3}$
}

\begin{abstract}
Objectives: The purpose of the current work is to evaluate D-dimer plasma level among both normal and complicated pregnancies such as gestational diabetes (GD) and hypertension (GH) among Saudi females. It is also focused on exhibiting any possible factors that could also play a role in rising D-dimer levels besides the age of the pregnant woman and the period of gestation.

Materials and Methods: A semi-quantitative latex agglutination assay was performed in the blood sample of healthy non-pregnant, pregnant and pregnant females with GD and GH.

Results: The results exhibited that $92 \%$ of pregnancies with GH and $90 \%$ of multiple pregnancies had high D-dimer levels. The analysis of the effect of age of pregnant women on D-dimer concentrations presented that $100 \%$ of pregnant women with GD, GH or multiple pregnancies aged above 40, had high levels of D-dimer. The results of the relationship between gestational age of pregnancies and $\mathrm{D}$-dimer showed that by reaching the third trimester, about $66.6 \%$ of women who had multiple pregnancies had $\mathrm{D}$-dimer concentration above $2000 \mathrm{ng} / \mathrm{mL}$. Interestingly, all the pregnant females with GH had D-dimer concentrations above 2000 $\mathrm{ng} / \mathrm{mL}$.

Conclusions: This study postulated that the concentrations of D-dimer significantly increase during pregnancy associated with common complications such as GD and GH. It is the first study to demonstrate the possible risk factors during pregnancy, which include age, number of pregnancies, gestational age and pregnancies with GD and GH, especially among Saudi Arabian women. Keywords: D-dimer, Pregnant, Complicated, Gestational diabetes, Hypertension, Saudi Arabia
\end{abstract}

\section{Introduction}

D-dimer is a minor protein product, which is found in circulation in negligible amounts because of endogenous fibrinolysis $(1,2)$. The normal or negative (decreased level) D-dimer result could exclude the serious intravascular thrombotic conditions, while the positive result (increased level) can be an indicator for the presence of an acute disorder related to abnormal clot formation such as venous thromboembolism (VTE). However, the site and/ or cause of the abnormal blood clot cannot be recognized by this test (3).

High D-dimer plasma level was reported during normal pregnancy and was described with proportional increase with gestational age as a result of enhancing maternal coagulation and increase the production of intrinsic and extrinsic coagulation factors $(4,5)$. During pregnancy, females with elevated concentrations of D-dimer are required to have further testing to exclude suspected VTE (6). A very recent study by Goodacre et al in 2018 demonstrated that D-dimer test must not be used for clinical examination of pulmonary embolism (PE) in pregnancy (7). However, another recent study by Choi et al in 2018 showed that the normal D-dimer results could be useful in excluding PE during pregnancy and postpartum patients. They showed that $\mathrm{D}$-dimer level was above 0.5 $\mathrm{mg} / \mathrm{L}$ (i.e. normal level) in all patients with PE (8).

In 2004, it has been found that D-dimers level significantly increases at 16 weeks of gestation (9). Another study was performed on healthy pregnant women and showed an increase in D-dimer level with each trimester (10). Moreover, D-dimer level was found to be significantly high among pregnant women with complicated disorders such as diabetes, preeclampsia, and abruption placentae $(11,12)$. A study by Manolov et al reported that during the third trimester of pregnancy, the preeclampsia development is correlated with the increased value of D-dimer compared with regular pregnancy (12). A significant increase in the level of $\mathrm{D}$-dimer was also seen during the pregnancy complicated with diabetes compared to uncomplicated third trimester pregnancies (11).

The current study was keen to estimate the plasma level of D-dimer in both normal and complicated pregnancies in Saudi pregnant women who attended the gynecology clinic in the medical center in Makkah region. This study was the first to be performed among Saudi females in the Western region of the Kingdom. 


\section{Materials and Methods}

Study Population

Ten healthy females who were non-pregnant, 24 females with normal pregnancy as well as 33 females with complicated pregnancy were involved in this study. Participants aged 19 to 50 years. Women with a risk of increased D-dimer level due to personal or family history of bleeding or thrombosis were excluded from this study.

\section{Samples}

Blue-top tube (Sodium citrate anticoagulant) was used to collect blood by a qualified lab specialist. Citrated plasma was obtained after one centrifugation at $1500 \mathrm{X}$ $\mathrm{g}$ for 15 minutes. The concentration of plasma D-dimer was measured using a Manual D-Dimer kit (Helena Biosciences Europe, UK) which is a semi-quantitative latex agglutination assay. Testing was completed within 2 hours of the blood draw in the medical center laboratory.

\section{Qualitative D-dimer}

Twenty microliters of patient plasma for positive and negative control were placed in the circle of the test card, an equal amount of the manual D-Dimer latex was added and mixed in each circle. The agglutination was read following 180 to 200 seconds and the results were compared to the controls. A positive result was recorded when agglutinated latex appeared, while non-agglutinated latex indicated a negative result.

\section{Quantitative D-dimer Concentration}

In case of positive result, serially diluted sample was prepared (1:2, 1:4 and 1:8). The concentration of the D-dimer can be determined from the reference values (Table 1).

\section{Statistical Analysis}

The data were manually entered into Microsoft Office Excel and were analyzed by Statistical Package of Social Sciences (SPSS), version 16.0.

\section{Results}

First, this study focused on evaluating and confirming that the D-dimer levels would increase in normal pregnancy than in non-pregnancy state. The results showed that there was an elevation in the D-dimer values in the healthily pregnant women in all the blood samples tested for this study group (Figure 1A). However, the D-dimer concentrations also dramatically increased compared to

Table 1. D-dimer Levels of Different Dilutions According to D-dimer Assay Kit

\begin{tabular}{lc}
\hline Dilution & Value $(\mathrm{ng} / \mathrm{mL})$ \\
\hline $1: 2$ & $250-500$ \\
$1: 4$ & $1000-2000$ \\
$1: 8$ & $>2000$ \\
\hline
\end{tabular}

the normal non-pregnant females included in the current research $(P<0.05)$ (Figure $1 \mathrm{~A})$. About $54 \%$ of the healthy pregnant women had high levels of D-dimer compared to only $20 \%$ of healthy non-pregnant women.

Regarding the blood samples taken from the women with complicated pregnancies, the results showed that the levels of D-dimer had no significant differences among the pregnant women with diabetes mellitus (DM). The results showed that about $45 \%$ of the women with DM had high D-dimer levels and about 54\% had normal D-dimer levels. Interestingly, the level of $\mathrm{D}$-dimer substantially increased in pregnant females with gestational hypertension $(\mathrm{GH})$ $(P<0.05)$ (Figure 1B). The results showed that about of $92 \%$ of the blood samples taken from the pregnant women with $\mathrm{GH}$ had high $\mathrm{D}$-dimer levels compared to only $8 \%$ who had regular D-dimer values (below $250 \mathrm{ng} / \mathrm{mL}$, based on the reference range kit used in the $\mathrm{D}$-dimer test) (Figure 1B). However, by investigating the $\mathrm{D}$-dimer levels of the blood samples of pregnancies with various complications (such as having allergies, a cough, a bypass operation, joint pain, anaemia and the common cold), we found that there
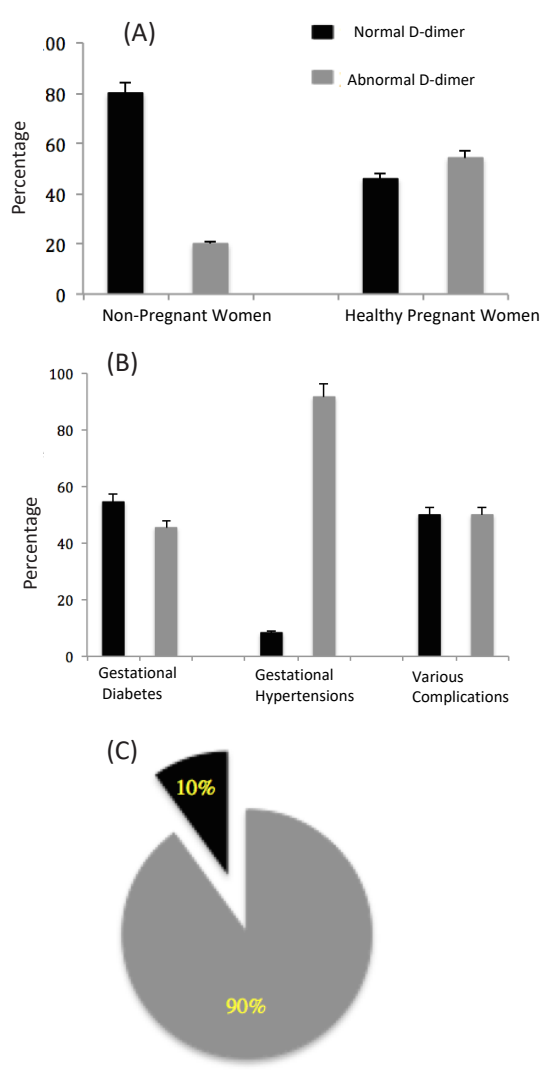

Figure 1. Evaluating of D-dimer Concentrations Among Healthy Pregnant Females and Pregnant Women with Common Complications. (A) Increased D-dimer levels in pregnant compared to non-pregnant. (B) The level of D-dimer in GD, hypertension and various complications (i.e. allergies, a cough, a bypass operation, joint pain, anaemia and the common cold) showed a considerable increase with the presence of Hypertension. (C) Elevated D-dimer levels and multiple pregnancies: $90 \%$ of women who had multiple pregnancies had very high (above normal) levels of $\mathrm{D}$-dimer and only $10 \%$ had normal D-dimer levels $(P<0.05)$. 
were insignificant differences among $\mathrm{D}$-dimer levels. The results showed about $50 \%$ of all the samples were positive and had high D-dimer levels (Figure 1B).

However, by comparing D-dimer levels in pregnant with $\mathrm{GH}$ to those who had either DM or any of the other acute illnesses listed above, the results also revealed a dramatic rise in values of $\mathrm{D}$-dimer in the pregnant women with $\mathrm{GH}$ (Figure 1B).

The levels of $\mathrm{D}$-dimer were also tested in blood samples from females who had multiple pregnancies. The results showed that $90 \%$ of these women had very high (above normal) D-dimer concentrations, and only $10 \%$ had normal D-dimer levels $(P<0.05)$ (Figure $1 C)$.

The levels of D-dimer in pregnant females with DM were also investigated according to their age. Accordingly, the blood samples were separated into categories by age (20-29 years old, 30-39 years old and 40-49 years old) to see if the age of the pregnant women would affect the levels of D-dimer. The results showed that the values of D-dimer elevated with age in the pregnant women. For the pregnant women aged 40 and above with DM, 100\% of them had high D-dimer levels, compared to $44 \%$ and $43 \%$ of the pregnant women aged between 20 and 30 years old. Nevertheless, there were insignificant differences in the levels of $\mathrm{D}$-dimer among the above-mentioned women between 20 and 30 years of age (Figure 2A).

In pregnant women with $\mathrm{GH}$, the results presented that the age of these women also affected the levels of D-dimer in their bodies. The results showed that $100 \%$ of the blood samples from these women had elevated D-dimer concentrations, with the concentrations increasing with their age. Women between the ages of 30 and 39 and 40 and 49 had higher D-dimer levels compared to women between the ages of 20 and 29 (Figure 2B).

This study also recorded concentrations of D-dimer in women who had multiple pregnancies, while still recording the impact of age on concentrations of D-dimer. The results showed that $100 \%$ of the blood samples taken from the women between the ages of 30 and 39 and between 40 and 49 had higher levels of D-dimer, compared to only $50 \%$ of the women who had experienced multiple pregnancies aged between 20 and 29 (Figure 2C).

An investigation of the D-dimer concentration for each trimester of pregnancy showed that about $75 \%$ of the women were placed within the regular value of $\mathrm{D}$-dimer concentrations in the first trimester (i.e. below $250 \mathrm{ng} /$ $\mathrm{mL}$ ), according to the D-dimer agglutination kit used in this study. In the second trimester of pregnancy, the results showed that $66 \%$ of these women had 1000 to $2000 \mathrm{ng} / \mathrm{mL}$ D-dimer concentrations and 33\% had normal D-dimer range. The ranges fluctuated between below $250 \mathrm{ng} /$ $\mathrm{mL}$ and $2000 \mathrm{ng} / \mathrm{mL}$ in the third trimester of pregnancy (Figure 3A).

Remarkably, by studying the gestational stages of pregnant women with hypertension, the results showed that $50 \%$ of these women had D-dimer concentrations of
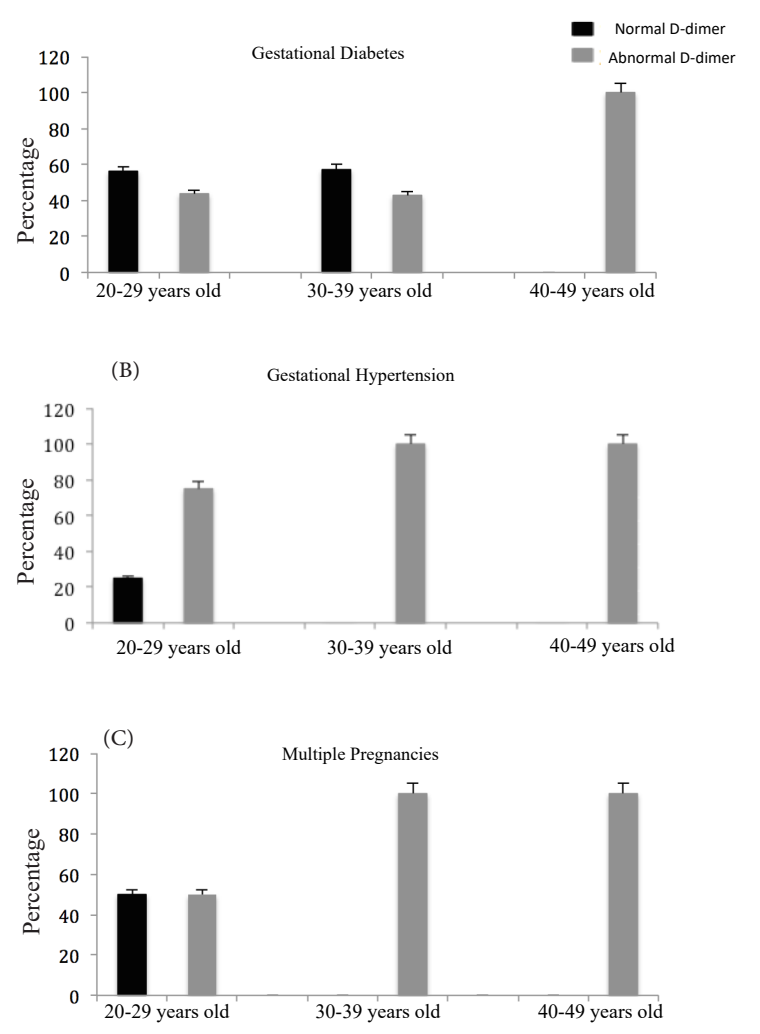

Figure 2. The Effect of the Age of Pregnant Women With Common Complications on Significant Increases in D-dimer Levels. (A) An analysis of D-dimer in pregnancy with GD showed that $100 \%$ of the pregnant women aged 40 and above with DM had high levels of D-dimer, compared to $44 \%$ and $43 \%$ of the pregnant women aged between 20 and 30 years old. (B) D-dimer was extensively elevated in the pregnancy with $\mathrm{GH}$ : women between the ages of 30 and 39 and 40 and 49 had higher levels of D-dimer (i.e. 100\%) compared to women between the ages of 20 and 29 (i.e. $50 \%$ ). (C) The effect of multiple pregnancies on D-dimer concentrations: the results showed that $100 \%$ of the blood samples taken from the women between the ages of 30 and 39 and between 40 and 49 had higher levels of D-dimer, compared to only $50 \%$ of the women who had experienced multiple pregnancies aged between 20 and 29 .

less than $250 \mathrm{ng} / \mathrm{mL}$ and $50 \%$ had D-dimer concentrations of more than $2000 \mathrm{ng} / \mathrm{mL}$ in the first trimester of pregnancy. The percentages of these women in the second trimester were distributed between very high $\mathrm{D}$-dimer concentrations, ranged between $1000 \mathrm{ng} / \mathrm{mL}$ and $2000 \mathrm{ng} /$ $\mathrm{mL}$, and D-dimer concentrations of above $2000 \mathrm{ng} / \mathrm{mL}$. Upon reaching the third trimester, all the women with $\mathrm{GH}$ had D-dimer levels above $2000 \mathrm{ng} / \mathrm{mL}$ (Figure 3B). In the third trimester, the concentrations of $\mathrm{D}$-dimer increased considerably, compared to the first and second trimester of the pregnancy $(P<0.05)$.

The number of pregnancies each woman had was also studied for $\mathrm{D}$-dimer concentrations by pregnancy trimester. The results showed that the concentrations of $\mathrm{D}$-dimer in the second trimester varied between less than $250 \mathrm{ng} / \mathrm{mL}$ and above $2000 \mathrm{ng} / \mathrm{mL}$. However, about $66.6 \%$ of the women had concentration above $2,000 \mathrm{ng} /$ $\mathrm{mL}$ in the third trimester, compared to the $33.3 \%$ of the women who had D-dimer concentration between 1000 $\mathrm{ng} / \mathrm{mL}$ and $2000 \mathrm{ng} / \mathrm{mL}$. The concentrations of D-dimer 
increased notably in the third trimester, compared to the second trimester of the pregnancy $(P<0.05)$ (Figure 3C).

\section{Discussion}

D-dimer is an outcome of the plasmatic breakdown of cross-linked fibrin, and it is commonly utilized as an index for the elimination VTE (13). Numerous studies have examined the elevation of D-dimer during pregnancy $(14,15)$ and illustrated that the amounts of D-dimer amplified during gestation compared to nongestation state. Moreover, increased plasmatic D-dimer levels were seen in the third trimester of the normal pregnancy (15). However, the reason behind the elevated D-dimer amounts during pregnancy could be due to the incessant coagulation and fibrinolysis increases the production of intrinsic and extrinsic coagulation factors during the normal placental development $(4,5)$. This leads to physiologically increased concentration of $\mathrm{D}$-dimer in maternal blood during the gestational period until delivery. This is not necessarily associated with any thromboembolic complications (16-18). Therefore, the current research is keen to investigate the amount of increased D-dimer levels throughout normal pregnancies, and, most importantly, in pregnancies with common complications, such as DM and hypertension among Saudi female. This study also focused on presenting and linking any possible factors that could also play a role in increasing concentrations of D-dimer besides the age of the pregnant woman and the period of gestation.

In this study, by comparing $\mathrm{D}$-dimer levels in normal pregnant females to normal non-pregnant females, we found that the D-dimer concentrations were considerably greater among the healthy pregnant women than the healthy non-pregnant women $(P<0.05)$. The results showed about $54.5 \%$ of the healthy pregnant and $20 \%$ of healthy non-pregnant women enrolled had high levels of D-dimer. This finding validated the previously studied evolutions of D-dimer values during pregnancy by Edelstam et al and Choi and Pai $(14,15)$.

Despite there being no previous studies about the connection between the increased levels of $\mathrm{D}$-dimer during healthy pregnancies and pregnancies with gestational diabetes (GD), our study spotted in this research area in order to find out if there is any association between D-dimer and pregnancy with GD. However, even with over eleven blood samples of pregnant women with GD included in this study, we found that about $45 \%$ of the women with DM had high D-dimer concentrations, and about 54\% had normal D-dimer concentrations. Nevertheless, these were not statistically significant differences $(P>0.05)$. More samples should be included to see if there are any more differences in the concentrations of D-dimer among pregnant females with DM. However, the current study suggests that GD could be considered as a factor in alterations in the concentrations of $\mathrm{D}$-dimer during pregnancy.
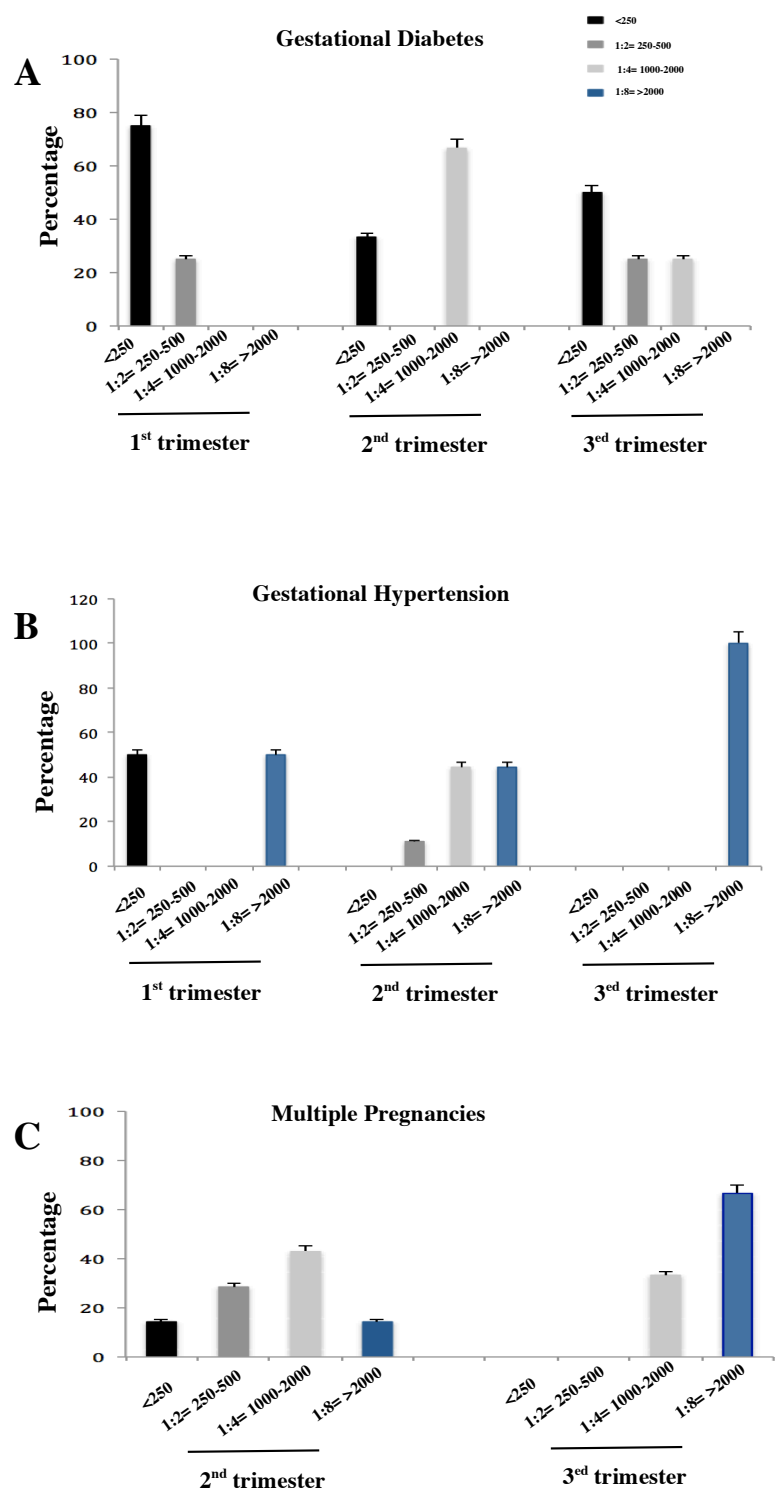

Figure 3. The Correlation of D-dimer Levels with Gestational Diabetes, Hypertension, Multiple Pregnancies and Gestational Age. (A) An analysis of the D-dimer concentration in pregnancy with GD showed that about $75 \%$ of the women had slandered D-dimer values in the first trimester (i.e. below $250 \mathrm{ng} / \mathrm{mL}$ ). Moreover, the results showed that $66 \%$ of these women had 1000 to $2000 \mathrm{ng} / \mathrm{mL}$ D-dimer concentrations and $33 \%$ had a normal range (i.e. below $250 \mathrm{ng} / \mathrm{mL}$ ) in the second trimester of pregnancy. The ranges fluctuated between below $250 \mathrm{ng} / \mathrm{mL}$ and $2000 \mathrm{ng} / \mathrm{mL}$ in the third trimester of pregnancy. (B) An extensive elevation was observed in the concentrations of D-dimer as the gestational age increased in the pregnant women with hypertension. The results exhibited that during the first trimester of pregnancy, about $50 \%$ of these women had D-dimer concentrations below $250 \mathrm{ng} / \mathrm{mL}$ and $50 \%$ had D-dimer concentrations of more than $2000 \mathrm{ng} / \mathrm{mL}$. For the second trimester, the percentages of these women were distributed between very high D-dimer concentrations, ranging between $1000 \mathrm{ng} /$ $\mathrm{mL}$ and $2000 \mathrm{ng} / \mathrm{mL}$, and D-dimer levels of over 2000. Upon reaching the third trimester, all the women with $\mathrm{GH}$ had D-dimer levels over $2000 \mathrm{ng} / \mathrm{mL}$. (C) Increased D-dimer concentrations as gestational age progresses could be associated with multiple pregnancies. The results showed that during the second trimester, D-dimer levels varied between less than $250 \mathrm{ng} / \mathrm{mL}$ and above $2000 \mathrm{ng} / \mathrm{mL}$. However, in the third trimester, about $66.6 \%$ of the women had D-dimer levels above 2000 $\mathrm{ng} / \mathrm{mL}$ compared to the $33.3 \%$ of the females who had D-dimer levels between $1000 \mathrm{ng} / \mathrm{mL}$ and $2000 \mathrm{ng} / \mathrm{mL}$. 
Interestingly, an analysis of D-dimer values in pregnancy with $\mathrm{GH}$ showed a dramatic rise in $\mathrm{D}$-dimer amounts. The results showed that about $92 \%$ of the blood samples from these females had high $\mathrm{D}$-dimer levels, compared to only $8 \%$ of the females who had standard D-dimer levels (below $250 \mathrm{ng} / \mathrm{mL}$, based on the reference range for kit used for the $\mathrm{D}$-dimer test). Our results agreed with the recent study by Kim et al in 2017, which investigated the clinical importance of D-dimer amounts in $\mathrm{GH}$ patients according to the severity of the disease. They stated that $80 \%$ of patients with GH who enrolled in their study had substantial elevations in the concentration of D-dimer (19). They also uncovered that the average of $\mathrm{D}$-dimer concentration was notably greater in patients with severe GH than in those with nonsevere GH (19). Nevertheless, due to the variances in the averages of $\mathrm{D}$-dimer concentrations among females with severe $\mathrm{GH}$ and non-severe GH, D-dimer cannot be used as an indicator or screening tool for identifying severe $\mathrm{GH}$. Moreover, as the severity of GH increased with each gestational stage, the substantial increase in $\mathrm{D}$-dimer in these women was also noted (19). However, they failed to investigate the $\mathrm{D}$-dimer concentrations during pregnancy in relation to the gestational period for comparison. Collectively, the current research confirmed that $\mathrm{GH}$ is a possible cause in increased $\mathrm{D}$-dimer levels, along with each stage of pregnancy. Therefore, more precautions should be implemented for pregnancies with GH, such as controlling high blood pressure in order to prevent damage to the mothers' kidneys and prevent the increased risk of preeclampsia or low birth weight. Moreover, the current study suggested that D-dimer could be helpful in early diagnoses and follow-ups for coagulopathy in cases of pregnancy-induced hypertension.

Another important factor taken into consideration in this study was gravidity (the number of times a woman had been pregnant), specifically in the Saudi Arabian population. Additionally, it has been published that parity or gravidity may be correlated with an enhanced probability of cardiovascular disorders. This may be produced due to childbearing history that could increase the levels of blood glucose and lipid (20). A study by Ness et al showed that having 6 or more pregnancies was correlated with the increase of cardiovascular disorder using 2 prospective American studies (20). Therefore, multiple pregnancies were one of the factors that we thought were related to increased D-dimer levels. Our study determined that $90 \%$ of women who had multiple pregnancies had higher D-dimer levels, compared to only $10 \%$ of women who had multiple pregnancies that had normal levels of D-dimer. This data concluded that the presence of elevated D-dimer amounts could also be associated with the number of pregnancies. Gravidity might be considered as an essential risk factor associated with new pregnancies. Further recommendations before planning new pregnancies would help to reduce the concentration of $\mathrm{D}$-dimer in these women, thereby creating a healthy pregnancy.

The age of pregnant women can also pose a challenge for healthy pregnancies. It is well known that older women who become pregnant are more likely to have common complications during their pregnancy, such as diabetes and high blood pressure. The inability to control these conditions makes these kinds of pregnancies even more complicated, which can contribute to miscarriages, poor fetal growth, and birth defects $(21,22)$. It has been established that the prevalence of VTD is increased with age, along with other major risk factors, for example, immobility, pregnancy, surgery, trauma, hormone use, obesity, and cancer. A four-month study by Alnomasy et al in 2012 that evaluated the levels of D-dimer among mature patients in the Al-Quwayyah Government hospital in Saudi Arabia found that the age of the patients played an essential part in increased D-dimer levels (23). In addition, a study by Tita-Nwa et al in 2010 also showed that the $\mathrm{D}$-dimer concentrations rise with age (24). All the above-mentioned studies linked the age of patients with elevated levels of D-dimer, however, our study focused on assessing the impact of age on D-dimer concentrations among healthy pregnancies, pregnancies with common complications, and other factors for the first time, particularly among pregnant Saudi Arabian women. Accordingly, we hypothesize that D-dimer levels could also increase with age in pregnant women.

In this study, we considered the pregnant women's age along with the various complications associated with their pregnancies. For GD complications, although there were insignificant differences between normal pregnancy and complicated pregnancy with GD, the results showed that $100 \%$ of pregnant women diagnosed with GD at the age of 40 or above had high D-dimer levels, compared to $44 \%$ and $43 \%$ of the pregnant women with GD aged between 20 and 30. These results signified that the age of the pregnant women, along with GD, would increase the possibility of high levels of D-dimer in their blood.

Our results showed that $100 \%$ of blood samples from these women had high D-dimer levels, with even higher levels in women aged between 30 and 39 and 40 and 49 , compared to women aged between 20 and 29. These results presented that the age of these women also affected the increase in D-dimer levels.

This study, along with the above-mentioned studies, confirmed that the age of the patients could increase the possibility of having increased $\mathrm{D}$-dimer levels. In particular, our results showed for the first time that the age of a pregnant woman could elevate $\mathrm{D}$-dimer concentration in her blood throughout her pregnancy. More specifically, our study correlated increased D-dimer concentrations with the ages of the pregnant women, the complications experienced during their pregnancies (i.e. GD and $\mathrm{GH}$ ) and the number of pregnancies experienced. The explanation behind increasing concentrations of $\mathrm{D}$-dimer with age could be due to rising lipid malformations, 
obesity and anaemia (24). These factors specify D-dimer concentrations as an indicative tool for thromboembolism in older people, particularly during pregnancy. Thus, more advice and recommendations should be given to help these patients during their pregnancy.

As mentioned earlier, the D-dimer concentration increased progressively throughout the pregnancy period. It has been established that, as the second trimester begins, the $\mathrm{D}$-dimer concentration will exceed the normal reference range and will be above $0.50 \mathrm{mg} / \mathrm{L}$ in more than half of pregnant women. This is also experienced by more than $90 \%$ of women in the third trimester (10). A prospective study by Kovac et al in 2010 investigated the D-dimer concentrations throughout pregnancy trimesters using a D-dimer analysis (i.e. quantitative latex immunoagglutination), with a value range of $230 \mathrm{ng} / \mathrm{mL}$. They found that $84 \%$ of all the women enrolled in their study had normal D-dimer levels in the first trimester, about $33 \%$ and only $1 \%$ in the second and third trimester, respectively. In addition, they found that the average of D-dimer levels in the first, second and third trimester was as follows: $222 \mathrm{ng} / \mathrm{mL}, 326 \mathrm{ng} / \mathrm{mL}$ and $475 \mathrm{ng} / \mathrm{mL}$. Therefore, they demonstrated an increase of D-dimer concentration of about $46 \%$, from 12 to 24 weeks and again from 24 to 34 weeks of gestation (25).

In our study, we found that $75 \%$ of pregnant women with GD had a normal reference range (i.e. below $250 \mathrm{ng} /$ $\mathrm{mL}$ ) of D-dimer levels. In the second trimester, the results showed that about $66 \%$ of these women had D-dimer concentration between $1000 \mathrm{ng} / \mathrm{mL}$ and $2000 \mathrm{ng} / \mathrm{mL}$, and the range fluctuated between below $250 \mathrm{ng} / \mathrm{mL}$ and between $250 \mathrm{ng} / \mathrm{mL}$ and $500 \mathrm{ng} / \mathrm{mL}$. D-dimer levels fluctuated between $1000 \mathrm{ng} / \mathrm{mL}$ and $2000 \mathrm{ng} / \mathrm{mL}$ in the third trimester. These results showed that, as gestational age increased, D-dimer levels also increased. However, our study is in consistent with the earlier studies that showed D-dimer concentration increases with each trimester of pregnancy.

The analysis of the gestational period of pregnant females with GH showed that $50 \%$ of these women had D-dimer concentration less than $250 \mathrm{ng} / \mathrm{mL}$ and that the other $50 \%$ D-dimer concentration of over $2000 \mathrm{ng} / \mathrm{mL}$ in the first trimester. For the second trimester, the percentage of pregnant women with GH was mainly categorized as very high $\mathrm{D}$-dimer concentration, fluctuated between $1000 \mathrm{ng} / \mathrm{mL}$ and $2000 \mathrm{ng} / \mathrm{mL}$ and D-dimer concentration above $2000 \mathrm{ng} / \mathrm{mL}$. Extraordinarily, upon reaching the third trimester, all the pregnant females with $\mathrm{GH}$ had D-dimer concentration over $2000 \mathrm{ng} / \mathrm{mL}$. These levels were significantly higher compared to their second trimesters $(P<0.05)$. Additionally, this data confirmed the significant role of $\mathrm{GH}$ conditions in the increase of $\mathrm{D}$-dimer concentration as the pregnancies progressed, even though high concentrations of $\mathrm{D}$-dimer were seen from the first trimester and continued to progress until delivery. This data, along with the above-mentioned data for pregnant women with $\mathrm{GH}$, showed that GH could be a signifier of high D-dimer levels during pregnancy. Women with $\mathrm{GH}$, who had multiple pregnancies, and each trimester of their pregnancies, were also studied for D-dimer levels. The results showed that the levels of D-dimer fluctuated between below $250 \mathrm{ng} / \mathrm{mL}$ and above $2000 \mathrm{ng} / \mathrm{mL}$ in the second trimester. However, about $66.6 \%$ of these women had D-dimer concentration exceeding $2000 \mathrm{ng} / \mathrm{mL}$ in the third trimester, compared to $33.3 \%$ of them having D-dimer concentration between $1000 \mathrm{ng} /$ $\mathrm{mL}$ and $2000 \mathrm{ng} / \mathrm{mL}$. The D-dimer levels meaningfully increased in the third trimester compared to the second trimester of the pregnancy $(P<0.05)$. All the abovementioned results agree with the previous studies which showed that increased plasmatic D-dimer levels were seen during the third trimester $(10,18,25)$. However, we confirmed that the increases in the levels $\mathrm{D}$-dimer were directly correlated with pregnancy complications.

In conclusion, the present study results showed that levels of D-dimer were notably higher in normal pregnancy compared to non-pregnancy situation. Additionally, during the second and third trimesters of pregnancy, the levels of D-dimer were more elevated than in the first trimester of pregnancy. These results were in agreement with the results stated in other previous studies. It is confirmed that concentrations of D-dimer increase during normal pregnancy, and, more importantly, that concentrations of D-dimer significantly increase in pregnancy associated with common complications. This work revealed for the first time that possible risk factors during pregnancy included age, number of pregnancies, gestational age and pregnancies with GD and GH. This study presented the correlation of these factors with a rise in the concentration of $\mathrm{D}$-dimer. In addition, our study is unique compared to previous studies because it demonstrated for the first time that $\mathrm{GH}$ and number of pregnancies can extraordinarily raise the D-dimer levels in Saudi Arabian pregnant females. Thus, more recommendations and advice should be given to women who are planning to become pregnant, particularly to those with a history of hypertension, to control the normal increase of D-dimer levels during pregnancy. This will prevent complications associated with high D-dimer levels during pregnancy. Nevertheless, a greater sample size would help correlate common pregnancy complications with D-dimer levels, specifically in samples of pregnancies with GD, as there are very few studies about it.

\section{Conflict of Interests}

Authors declare that they have no conflict of interests.

\section{Ethical Issues}

The Research Committee of the College of Applied Medical Sciences approved this study. 


\section{Financial Support}

This study did not receive any specific funding from grant agencies in the public, commercial, or charitable sectors.

\section{References}

1. Prisco D, Ciuti G, Falciani M. Hemostatic changes in normal pregnancy. Haematol Rep. 2005;1(10):1-5.

2. Jeremiah ZA, Adias TC, Opiah M, George SP, Mgbere O, Essien EJ. Elevation in D-dimer concentrations is positively correlated with gestation in normal uncomplicated pregnancy. Int J Womens Health. 2012;4:437-443. doi:10.2147/ijwh.s32655

3. Buseri FI, Jeremiah ZA, Kalio FG. Influence of pregnancy and gestation period on some coagulation parameters among Nigerian antenatal women. Res J Med Sci. 2008;2(6):275-281.

4. Giavarina D, Mezzena G, Dorizzi RM, Soffiati G. Reference interval of D-dimer in pregnant women. Clin Biochem. 2001;34(4):331-333.

5. Nishii A, Noda Y, Nemoto R, et al. Evaluation of D-dimer during pregnancy. J Obstet Gynaecol Res. 2009;35(4):689693. doi:10.1111/j.1447-0756.2008.01007.x

6. Brown MD, Lau J, Nelson RD, Kline JA. Turbidimetric D-dimer test in the diagnosis of pulmonary embolism: a metaanalysis. Clin Chem. 2003;49(11):1846-1853.

7. Goodacre S, Horspool K, Nelson-Piercy C, et al. The DiPEP study: an observational study of the diagnostic accuracy of clinical assessment, D-dimer and chest $\mathrm{x}$-ray for suspected pulmonary embolism in pregnancy and postpartum. BJOG. 2018. doi:10.1111/1471-0528.15286

8. Choi H, Krishnamoorthy D. The diagnostic utility of D-dimer and other clinical variables in pregnant and post-partum patients with suspected acute pulmonary embolism. Int J Emerg Med. 2018;11(1):10. doi:10.1186/ s12245-018-0169-8

9. Morse M. Establishing a normal range for D-dimer levels through pregnancy to aid in the diagnosis of pulmonary embolism and deep vein thrombosis. J Thromb Haemost. 2004;2(7):1202-1204. doi:10.1111/j.15387836.2004.00776.x

10. Kline JA, Williams GW, Hernandez-Nino J. D-dimer concentrations in normal pregnancy: new diagnostic thresholds are needed. Clin Chem. 2005;51(5):825-829. doi:10.1373/clinchem.2004.044883

11. Ballegeer V, Mombaerts P, Declerck PJ, Spitz B, Van Assche FA, Collen D. Fibrinolytic response to venous occlusion and fibrin fragment $\mathrm{D}$-dimer levels in normal and complicated pregnancy. Thromb Haemost. 1987;58(4):1030-1032.

12. Manolov V, Marinov B, Masseva A, Vasilev V. [Plasma D-dimer levels in preeclampsia]. Akush Ginekol (Sofiia). 2014;53 Suppl 2:15-18.

13. Reber G, Vissac AM, de Moerloose P, Bounameaux H,
Amiral J. A new, semi-quantitative and individual ELISA for rapid measurement of plasma D-dimer in patients suspected of pulmonary embolism. Blood Coagul Fibrinolysis. 1995;6(5):460-463.

14. Edelstam G, Lowbeer C, Kral G, Gustafsson SA, Venge P. New reference values for routine blood samples and human neutrophilic lipocalin during third-trimester pregnancy. Scand J Clin Lab Invest. 2001;61(8):583-592.

15. Choi JW, Pai SH. Tissue plasminogen activator levels change with plasma fibrinogen concentrations during pregnancy. Ann Hematol. 2002;81(11):611-615. doi:10.1007/s00277002-0549-1

16. Szecsi PB, Jorgensen M, Klajnbard A, Andersen MR, Colov NP, Stender S. Haemostatic reference intervals in pregnancy. Thromb Haemost. 2010;103(4):718-727. doi:10.1160/th0910-0704

17. Francalanci I, Comeglio P, Alessandrello Liotta A, et al. D-dimer plasma levels during normal pregnancy measured by specific ELISA. Int J Clin Lab Res. 1997;27(1):65-67.

18. Nolan TE, Smith RP, Devoe LD. Maternal plasma D-dimer levels in normal and complicated pregnancies. Obstet Gynecol. 1993;81(2):235-238.

19. Kim SJ, Ahn HJ, Park JY, et al. The clinical significance of D-dimer concentrations in patients with gestational hypertensive disorders according to the severity. Obstet Gynecol Sci. 2017;60(6):542-548. doi:10.5468/ ogs.2017.60.6.542

20. Ness RB, Harris T, Cobb J, et al. Number of pregnancies and the subsequent risk of cardiovascular disease. N Engl J Med. 1993;328(21):1528-1533. doi:10.1056/ nejm199305273282104

21. Viau PA, Padula CA, Eddy B. An exploration of health concerns \& health-promotion behaviors in pregnant women over age 35. MCN Am J Matern Child Nurs. 2002;27(6):328-334.

22. Maheshwari A, Porter M, Shetty A, Bhattacharya S. Women's awareness and perceptions of delay in childbearing. Fertil Steril. 2008;90(4):1036-1042. doi:10.1016/j. fertnstert.2007.07.1338

23. Alnomasy SF, Ansari S, Izhari MA, Alam S, Ali S. Evaluation of D-dimer level among adult patients in Al-Quwayyah Government Hospital, Saudi Arabia. J Appl Pharm Sci. 2012;2(12);82-84. doi:10.7324/JAPS.2012.21215.

24. Tita-Nwa F, Bos A, Adjei A, Ershler WB, Longo DL, Ferrucci L. Correlates of D-dimer in older persons. Aging Clin Exp Res. 2010;22(1):20-23.

25. Kovac M, Mikovic Z, Rakicevic L, et al. The use of D-dimer with new cutoff can be useful in diagnosis of venous thromboembolism in pregnancy. Eur J Obstet Gynecol Reprod Biol. 2010;148(1):27-30. doi:10.1016/j. ejogrb.2009.09.005

(C) 2019 The Author (s); This is an open-access article distributed under the terms of the Creative Commons Attribution License (http://creativecommons.org/licenses/by/4.0), which permits unrestricted use, distribution, and reproduction in any medium, provided the original work is properly cited. 